\title{
MICROFLUIDIC MICROALGAL BIOREACTOR FOR HIGH-THROUGHPUT SCREENING OF CO2 CONCENTRATION CONDITIONS ON MICROALGAE GROWTH
}

\author{
Zhen Xu, Yingjun Wang, Yuncong Chen, Martin H. Spalding, and Liang Dong* \\ Iowa State University, Ames, Iowa, USA
}

\begin{abstract}
This paper presents a high-throughput microfluidic bioreactor capable of culturing and screening microalgal strains under different $\mathrm{CO}_{2}$ envionmental conditions. The present bioreactor consists of a microfluidic microalgae culture unit, a $\mathrm{CO}_{2}$ concentration gradient generation (CGG) unit, and an in-line optical detection unit for quantifying cell growth. A gas semipermeable membrane is designed between the microfluidic culture and CGG units to control mass transport of $\mathrm{CO}_{2}$ into the cell culture channels. To validate the workability of this device, Chlamydomonas reinhardtii strain CC620 was cultured in the bioreactor and its growth rate under different $\mathrm{CO}_{2}$ concentrations was quantified. In-line monitoring of cell growth is achieved using both light intensity measurement and colorimetric detection. The present bioreactor system will have a great potential to accelerate microalgae research.
\end{abstract}

\section{INTRODUCTION}

Microalgae are a promising renewable energy source [1]. It is important to understand algal gene regulation and optimize growth conditions (e.g., light intensity and cycle, $\mathrm{CO}_{2}$, temperature, etc) for discovery of new strains [2]. Current microalgal studies are often conducted in shake flasks, open raceway ponds, and large size bioreactors [3-6]. However, considering the numerous microalgal strains and combinatorial nature of many growth factors, these conventional culture systems appear less suitable for rapid, large scale, high throughput screening and tracking microalgae growth responding to different environmental conditions. Also, due to large size of the conventional systems, it is relatively difficult to achieve spatially uniform environmental conditions inside these bioreactors without sufficient agitation by shaking, rotating, and/or bubbling. Furthermore, in-line monitoring and quantification of algal growth has rarely been achieved in the conventional systems.

Microfluidics has recently been widely researched and applied to enable miniaturization and automation of biochemical processes and analysis for many biological applications [7]. Cell culture on microfluidic devices has attracted much attention in the area of experimental cell biology. Microfluidic bioreactors have also been reported to screen microalgal oil production under different light intensity conditions, showing great promise of employing microfluidic technology to improve microalgae research [8-11]. In this paper, we report, for the first time, the development of a microfluidic algal bioreactor for high-throughput screening of $\mathrm{CO}_{2}$ conditions on microalgae growth. The microfluidic design of the system will bring about several benefits, including parallelizing multiple experiments of culturing microalgal cells under different $\mathrm{CO}_{2}$ conditions, exposing cells to uniformly spatially distributed $\mathrm{CO}_{2}$ inside each bioreactor, and automatically monitor cell growth with minimum labor.

\section{DEVICE DESIGN}

The microfluidic bioreactor is composed of a microfluidic cell culture unit, a gas concentration gradient generation (CGG) unit, and an optical detection unit for in-line monitoring microalgal growth (Fig. 1a). A gas semipermeable hydrophobic membrane is sandwiched by the culture unit and the CGG unit. This membrane holds the culture medium inside the upper growth channels and allows $\mathrm{CO}_{2}$ to diffuse from the lower gas channels into the culture medium through the built-in nanopores of the membrane (Fig. 1b). The diffused $\mathrm{CO}_{2}$ is used as carbon source to support photosynthesis of microalgae. To compensate for possible medium loss due to evaporation, two miniature source reservoirs are formed at the inlet and outlet of each culture channel and loaded with fresh pure growth medium. This allows for automatic refilling of the growth channel when any possible medium losses occur at the semipermeable membrane. The device allows for a number of advantages. First, mass transport of $\mathrm{CO}_{2}$ into cell culture medium is faster because of a small diffusion length. Second, spatial distribution of $\mathrm{CO}_{2}$ within each culture channel is uniform because the hydrophobic semipermeable membrane allows $\mathrm{CO}_{2}$ to diffuse into the channel evenly over the entire culture channel area. Third, different $\mathrm{CO}_{2}$ concentrations can be flexibly generated, facilitating the study of their influence on cell growth.
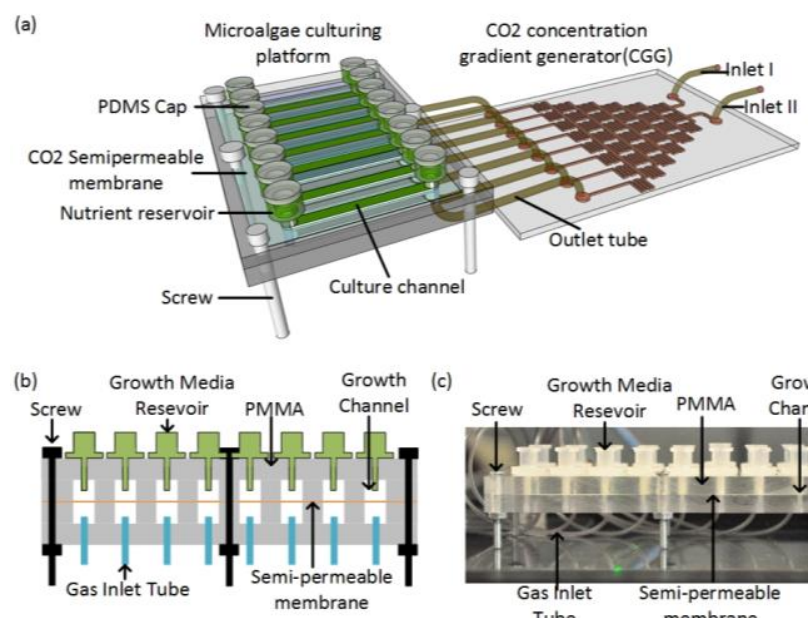

(d)
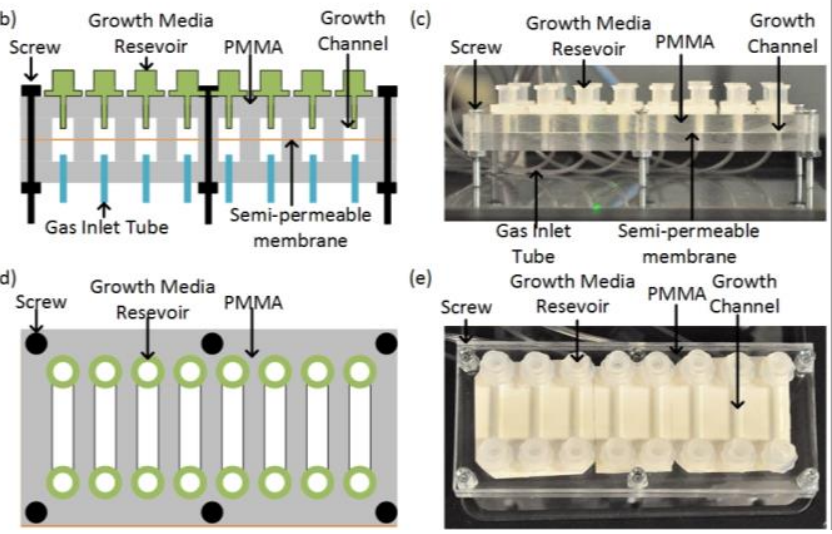

Figure 1: (a) Schematic of the proposed microalgal bioreactor. (b, c) and $(d, e)$ are the side view and the top view of a microfluidic cell culture device, respectively.

To realize in-line monitoring of cell growth, a transmitted light intensity (TLI) measurement setup is designed and interfaced with the cell culture device, where $532 \mathrm{~nm}$ wavelength laser is used to probe cell growth density. Generally, TLI decreases with increasing cell density. Time course measurement for all the culture channels is realized by placing the device on a programmable motorized stage. Besides, a simple colorimetric method is taken to estimate cell growth by taking pictures for all the growth channels using a digital camera and conducting gray-value analysis (GVA).
Solid-State Sensors, Actuators and Microsystems Workshop Hilton Head Island, South Carolina, June 5-9, 2016 


\section{ON-CHIP CELL CULTURE}

To demonstrate the workability of the proposed bioreactor, Chlamydomonas reinhardtii strain CC620, a wild green microalgae strain, was used as our model microalgae. Its growth under different $\mathrm{CO}_{2}$ conditions was characterized with the developed system. After loading $C$. reinhardtii strain into the growth channels, the miniature nutrient reservoirs were filled by none-carbon TAP medium and then sealed with polydimethylsiloxane or PDMS based plugs. The device was placed on the motorized stage for in-line TLI monitoring of cell growth. The cells were cultured at room temperature under a 9-watt fluorescent lamp. The distance between the lamp and the device was set to be $30 \mathrm{~cm}$.

Different $\mathrm{CO}_{2}$ concentration conditions were created using the CGG. To realize this, the two inlets of the CGG were respectively connected to a customized air cylinder with $50 \mathrm{ppm} \mathrm{CO}_{2}$ and a normal air cylinder with $300 \mathrm{ppm} \mathrm{CO}_{2}$. The flow rates of the input gas flows were controlled by two mass flow controllers. The eight outlets of the CGG unit were connected to the corresponding gas flow channels of the culture device.
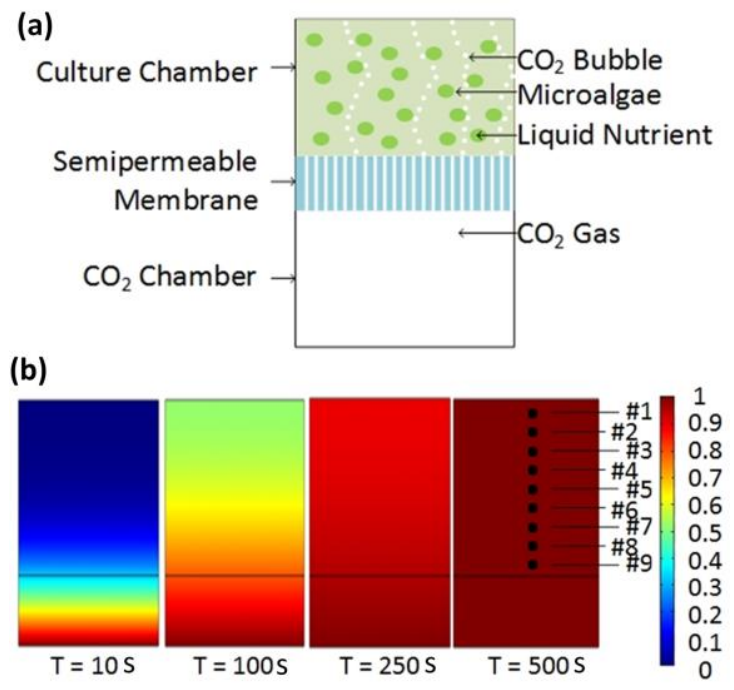

(c)

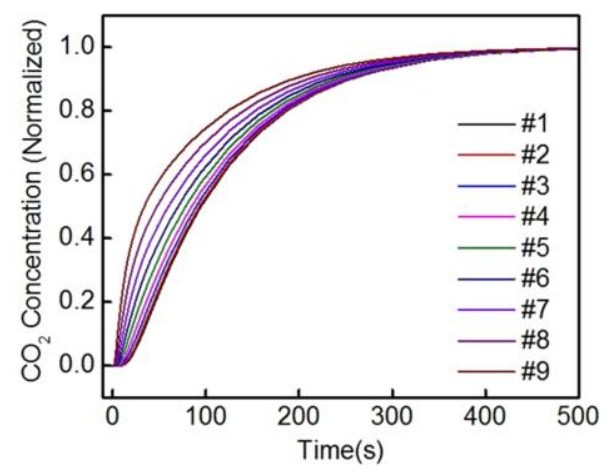

Figure 2: (a) Schematic (cross-section view) of a single cell culture channel. (b) Simulated $\mathrm{CO}_{2}$ distribution in a gas permeable hydrophobic membrane and the culture medium at $t=10,100,250$, and $500 \mathrm{s.}$. (c) Normalized $\mathrm{CO}_{2}$ concentrations at different locations along the height of the growth channel as a function of time. See the locations of \#1-\#9 in (b).

\section{RESULTS AND DISCUSSION}

Fluid dynamic simulations ware taken to study $\mathrm{CO}_{2}$ distribution in the gas permeable membrane and the culture medium using a finite element analysis (FEA) method based software (COMSOL). In this model, a single cell culture channel consists of three parts: a culture chamber with liquid medium, a semipermeable membrane, and a $\mathrm{CO}_{2}$ chamber (Fig. 2a). Fig. $2 \mathrm{~b}$ indicates the fast formation of a spatially uniform $\mathrm{CO}_{2}$ environment in the culture channel within $300 \mathrm{sec}$, without using any agitation methods such as shaking, rotating, and bubbling.

Fig. 3a shows the fabricated CGG. Fig. 3b shows the simulated and experimental results of $\mathrm{CO} 2$ concentrations at the outputs of the CGG. Here, FEA simulation was performed using the models of laminar flows and transport of diluted species. The generated $\mathrm{CO}_{2}$ concentrations were measured by a $\mathrm{CO}_{2}$ meter. The mean values for the measured concentrations at eight outputs of the CGG were 272 ppm, 232 ppm, 211 ppm, 190 ppm, 159 ppm, 118 ppm, 60 ppm, and $50 \mathrm{ppm}$, matching well with the simulation result (Fig. 3b).
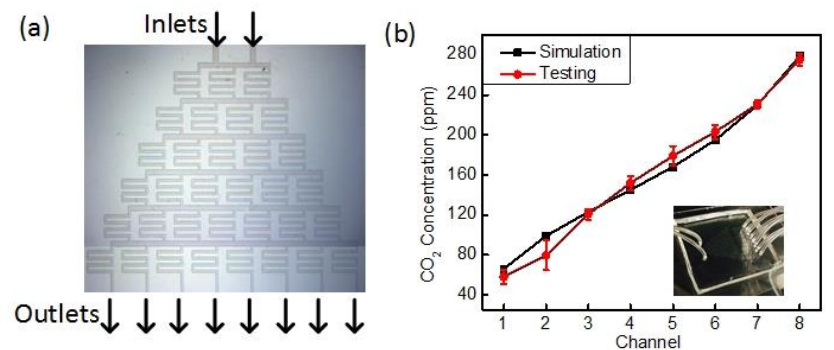

Figure 3: (a) Photo of a microfluidic CGG for generating eight different $\mathrm{CO}_{2}$ concentrations. (b) Simulated and measured $\mathrm{CO}_{2}$ concentrations at the outputs of the $C G G$.
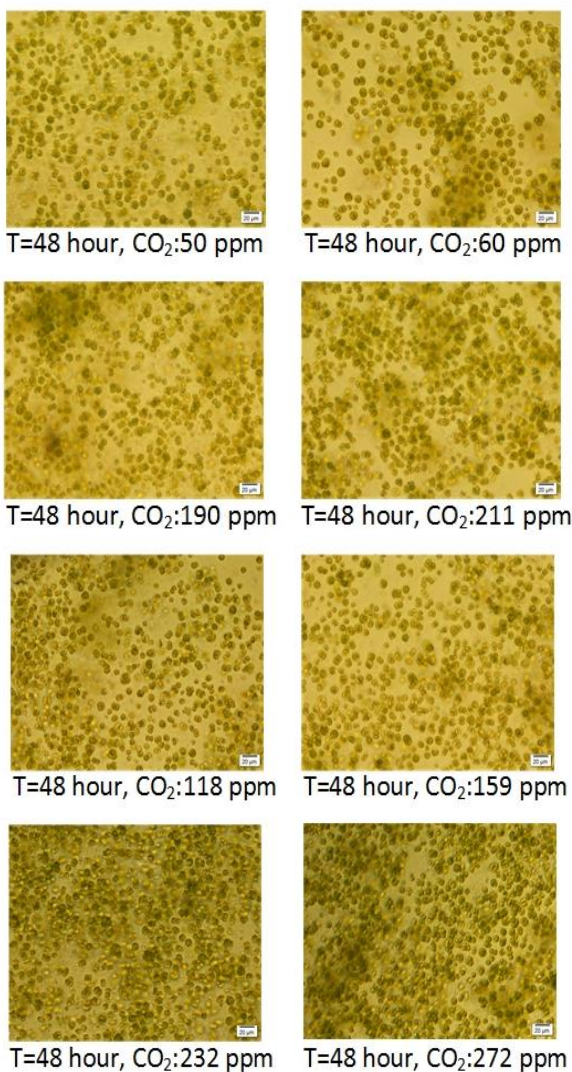

Figure 4: Microscopic photos of C. reinhardtii strain after growing in the channels for 48 hours under different $\mathrm{CO} 2$ conditions. 
(a)

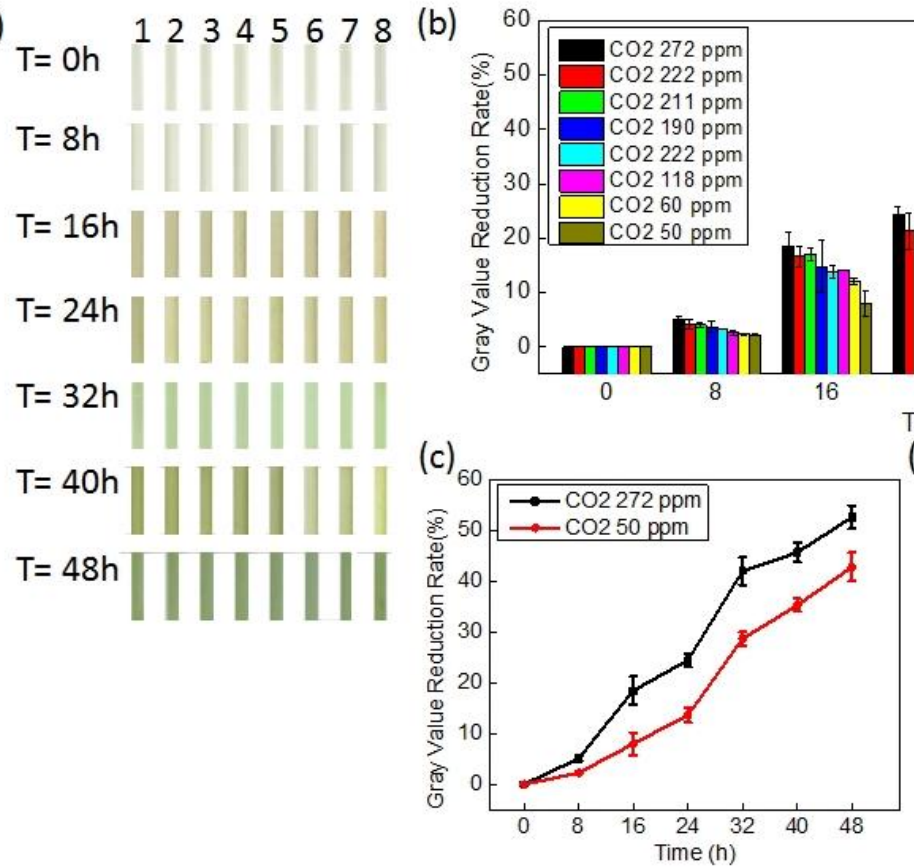

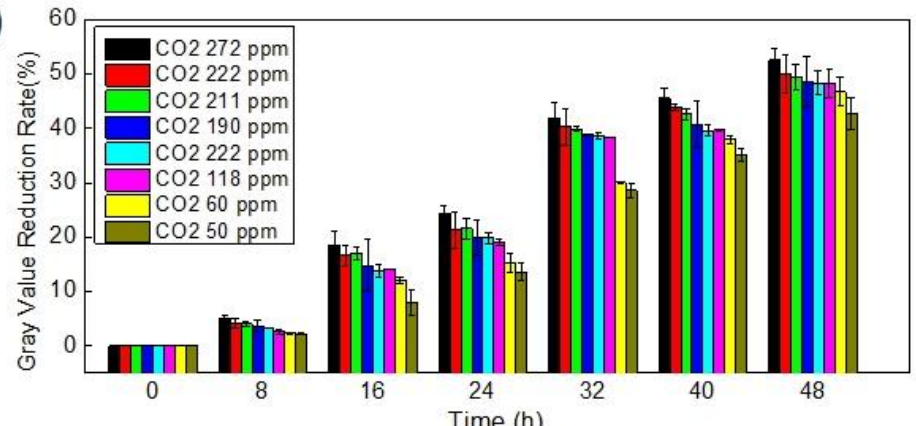

(d)

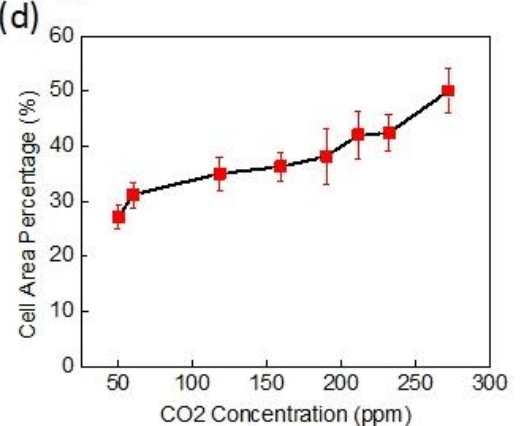

Figure 5: Analysis of C. reinhardtii growth under 8 different $\mathrm{CO}_{2}$ concentrations over 48 hours. (a) Growth images taken every 8 hours for all the channels at different $\mathrm{CO}_{2}$ conditions: $272 \mathrm{ppm}, 232 \mathrm{ppm}, 211 \mathrm{ppm}, 190 \mathrm{ppm}, 159 \mathrm{ppm}, 118 \mathrm{ppm}, 60 \mathrm{ppm}$, and $50 \mathrm{ppm}$. (b) Gray values for the growth images in all the channels over 48 hours. (c) Gray value reduction rate over 48 hours under $\mathrm{CO}_{2}$ conditions: 272 ppm and 50 ppm. (d) Strain cells area percentage under different $\mathrm{CO}_{2}$ concentrations after 48 hours.

(a)

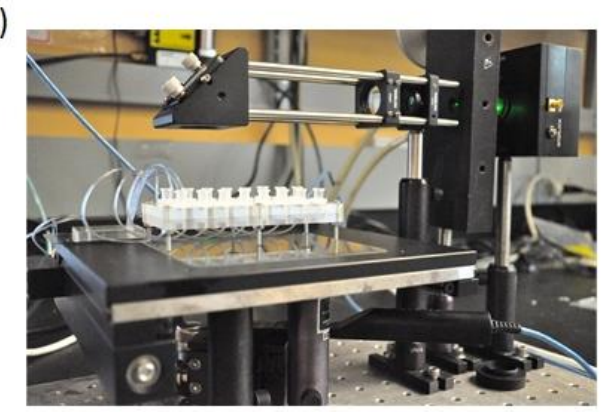

(b)

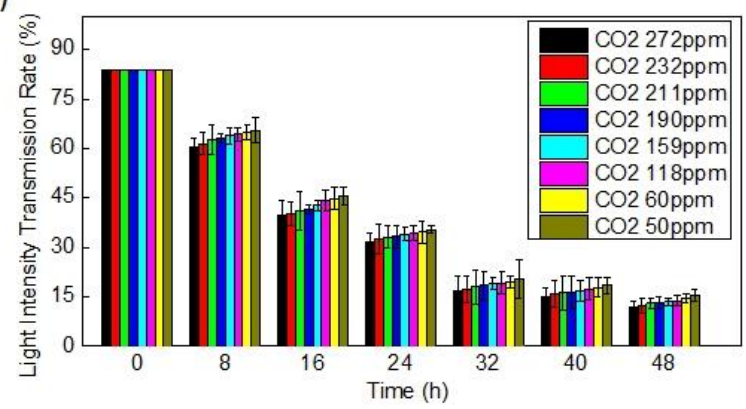

(c)

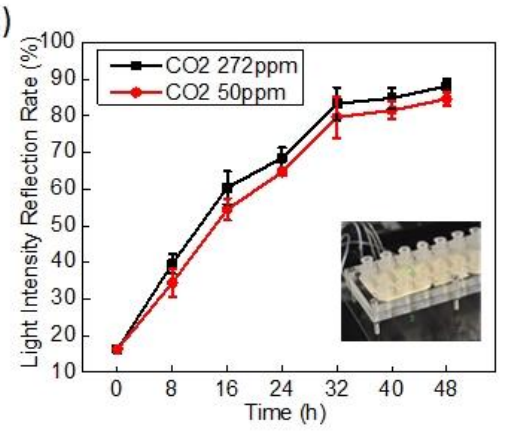

Figure 6: (a) Transmitted light intensity measurement setup. $532 \mathrm{~nm}$ wavelength laser is used. (b) Result of light intensity transmission under eight $\mathrm{CO}_{2}$ concentrations in 48 hours at different $\mathrm{CO}_{2}$ concentrations: 50, 60, 118, 159, 190, 211, 232, and 272 ppm. (c) Light intensity reflection rate under $\mathrm{CO}_{2}$ concentrations: $272 \mathrm{ppm}$ and $50 \mathrm{ppm}$. 
Fig. 4 shows the growth of $C$. reinhardtii at 48 hours inside the culture channels of the device under eight $\mathrm{CO}_{2}$ concentrations. It is obvious that the cell density increased differently, depending on the surrounding $\mathrm{CO}_{2}$ conditions. To better quantify this environmental impact, we characterized cell growth based on both the GVA using image analysis software (Image J) and the TLI measurement for each channel. Fig. 5a shows the growth images of $C$. reinhardtii at $0,8,16,24,32,40,48$ hours with different $\mathrm{CO}_{2}$ concentrations. Due to the given difference in $\mathrm{CO}_{2}$ supplies, the cell density increased at different rates. The higher the $\mathrm{CO}_{2}$ concentration, the higher the cell growth rate. Specifically, at 48 hours, the gray value of the cell growth image at $272 \mathrm{ppm}$ reduced to $52.5 \%$, while only $42.7 \%$ reduction in gray value was found at $50 \mathrm{ppm}$ (Fig. 5c). Another analysis was taken to obtain the percentage of the cell coverage area over the whole channel area after 48-hour culturing. The obtained highest coverage percentage was $50.1 \%$ at $272 \mathrm{ppm}$, compared to the lowest value of $27.19 \%$ at 50 ppm (Fig. $5 \mathrm{~d}$ ).

Fig. 6a shows the setup for the TLI measurement. The measured result (Fig. 6b-c) indicates a similar cell growth trend to the GVA result for different $\mathrm{CO}_{2}$ conditions. Also, the on-chip cell growth results agree well with the plate-based cell growth results, indicating that the present device technology can be suitably used in screening $\mathrm{CO}_{2}$ conditions for culturing different microalgal species and their strains.

Compared to conventional bioreactors, the volume of the microfluidic culture channel is only less than one milliliter. The small scale of the device will allow for a large number of growth conditions to be tested simultaneously, which is difficult to be done in large batch growth, as it is now utilized. More importantly, the miniaturized device with the gas permeable hydrophobic membrane allows for faster $\mathrm{CO}_{2}$ mass transportation, and uniform spatial $\mathrm{CO}_{2}$ concentration.

It should be also pointed out that current study is focused on studying the effect of $\mathrm{CO}_{2}$ conditions on growth of one single microalgae strain. With minor structural modifications, the microfluidic device will be used to investigate growth of multiple strains under the same environment. Furthermore, in the future, we will build an integrated bioreactor to simulate more natural microalgal growth environments with multiple controllable factors, including $\mathrm{pH}$, light condition, $\mathrm{pH}$ value, temperature, and nutrient.

\section{CONCLUSIONS}

A microfluidic microalgal bioreactor was developed to study the growth of microalgae under different $\mathrm{CO}_{2}$ concentrations in parallel. C. reinhardtii strain CC620 was characterized using the platform. The system simulated natural growth environment for microalgae and provided a capability of real-time screening, with higher throughput and lower cost than traditional conventional bioreactors. Further, the system could be extended to study other culture factors. It is believed that this system can work as a powerful tool to investigate how algal growth are influenced by environmental conditions and other culture factors at significantly lower cost and shorter time, which would help further development of biorenewable fuel.

\section{ACKNOWLEDGEMENTS}

This work was supported in part by the National Science Foundation under grants ECCS-0954765 and DBI-1353819, the Plant Sciences Institute of Iowa State University (ISU), and the ISU Crop Bioengineering Consortium.

\section{REFERENCES}

[1] Y. Chisti, "Biodiesel from microalgae," Biotechnol. Adv., 25, 294-306 (2007).
[2] Y. Chisti, "Biodiesel from microalgae beats bioethanol", Trends in Biotechnology, 26, 126-131 (2008).

[3] D.R. Georgianna and S.P. Mayfield, "Exploiting diversity and synthetic biology for the production of algal biofuels," Nature, 488, 329-335 (2012).

[4] A. Saeed and M. Iqbal, "Immobilization of blue green microalgae on loofa sponge to biosorb cadmium in repeated shake flask batch and continuous flow fixed bed column reactor system", World Journal of Microbiology \& Biotechnology,22, 775-782 (2006).

[5] S. Boussiba, E. Sandbank, G. Shelef, Z. Cohen, A. Vonshak, A. Benamotz, S. Arad and A. Richmond, "Outdoor cultivation of the marine microalga Isochrysis galbana in open reactors", Aquaculture, 72, 247-253 (1998).

[6] J. Degen, A. Uebele, A. Retze, U. Schmid-Staiger and W. Trosch, "A novel airlift photobioreactor with baffles for improved light utilization through the flashing light effect", Journal of Biotechnology, 92, 89-94 (2001).

[7] H. Jiang, Z. Xu, M. R. Aluru and L. Dong, "Plant chip for high-throughput phenotyping of Arabidopsis", Lab on a Chip, 14, 1281-1293 (2014).

[8] H. Kim, T.L. Weiss, H.R. Thapa, T.P. Devarenne and A. Han, "A microfluidic photobioreactor array demonstrating high-throughput screening for microalgal oil production," Lab on a Chip, 14,1415 (2014).

[9] R. A. Erickson and R. Jimenez, "Microfluidic cytometer for high-throughput measurement of photosynthetic characteristics and lipid accumulation in individual algal cells", Lab on a Chip, 13, 2893-2901 (2013).

[10] G. Zheng, Y. Wang, Z. Wang, W. Zhong, H. Wang and Y. Li, "An integrated microfluidic device in marine microalgae culture for toxicity screening application", Marine Pollution Bulletin, 72, 231-243 (2013).

[11] A. Dewan, J. Kim, R. H. McLean, S. A. Vanapalli and M. N. Karim, "Growth kinetics of microalgae in microfluidic static droplet arrays", Biotechnology and Bioengineering, 109, 2987-2996 (2012).

\section{CONTACT}

*Liang Dong, tel: +1-515-294-0388; ldong@iastate.edu 